\title{
Toward web accessibility for older users
}

\author{
Anna C. Cavender · Jeffrey P. Bigham
}

Published online: 30 January 2011

(c) Springer-Verlag 2011

Older adults are increasingly accessing the web for numerous reasons: searching for health and travel information, socializing with friends and family, or simply for entertainment. Accommodating and designing for the range of skills, experience, and physical and mental abilities of this user group can be a challenge. Although the causes may differ, commonalities are often found among older adults and people with disabilities in terms of perceptual, motor, and cognitive issues with web usage.

Web Accessibility for Older Users was the theme of the W4A (Web-4-All) conference in 2009 and, from a list of the many high-quality papers presented at the conference, the most relevant, innovative work was selected for extension in this special issue. This collection of articles addresses the growing need to better understand the current motivations, uses, and barriers for older adults in accessing the web, the subsequent design and improvement to accessibility guidelines to better include older adults, and the factors that have led to the current usage levels and the extent to which those factors will continue to effect technology usage for the next generation of older adults.

Understanding barriers that may hinder this unique and diverse population in accessing the web is an important first step. Three articles in this special issue further current knowledge of Internet use, common problems encountered, and factors that prevent access altogether for older adults.

A. C. Cavender ( $\square)$

Department of Computer Science and Engineering,

University of Washington, Seattle, WA, USA

e-mail: cavender@cs.washington.edu

\section{J. P. Bigham}

Department of Computer Science, University of Rochester, Rochester, NY, USA
"An ethnographical study of the accessibility barriers in the everyday interactions of older people with the web", by Sergio Sayago and Josep Blat, discusses a study of older people interacting with the web over 3 years. In this study, older adults were motivated to access the web to seek inclusion, independence, and socialization. Similarly, "A survey of technology accessibility problems faced by older users in China”, by Dengfeng Yao, Yunfeng Qiu, Harry Huang, Zaixin Du, and Jianqing Ma, investigates how the aging population in China uses the web and presents recommendations for lowering the barriers faced by this group. Focusing on a specific web service, "Designing accessible experiences for older users: User requirement analysis for a railway ticketing portal”, by Özge Subasi, Michael Leitner, Norman Hoeller, Arjan Geven, and Manfred Tscheligi, describes a study conducted with users of a ticketing portal at a train station. A number of design principles and changes to accessibility guidelines are recommended.

Current web accessibility guidelines do not yet explicitly consider older users, making it difficult to evaluate a web page or web site for its ability to reach this audience. Comprehensive guidelines for such a diverse population remain elusive, but two articles in this issue begin to fill this void. "Web accessibility guideline aggregation for older users and its validation”, by Giorgio Brajnik, Yeliz Yesilada, and Simon Harper, develops a new methodology for evaluating web accessibility for older users by using an aggregation of barrier types found for people with low vision and mobility impairments. "Validating the effectiveness of EvalAccess when applying WCAG 2.0 tests", by Amaia Aizpurua, Myriam Arrue, Markel Vigo, and Julio Abascal, presents an extension to EvalAccess, an evaluation tool for supporting WCAG 1.0 standard, to also include the WCAG 2.0 standard and then compares 
automatic evaluation using WCAG 1.0, WCAG 2.0, and manual evaluation.

The final paper, "Technology skill and age: What will be the same 20 years from now?" by Vicki Hanson, looks beyond today's technology and today's older population, and questions the difference between age-related and experience-related issues that affect users' ability to access new technology. In designing for this audience, Hanson recommends focusing not only on deficits and disabilities of older users but also on their strengths. The deliberate and focused approaches to search and careful decisions employed by older users are one example. Creating a clearer separation of barriers due to age-related issues and those due to inexperience will ensure a better understanding and generalizability of today's findings in the future.

The editors of this special issue would like to thank the Editorial Board of the International Journal Universal Access in the Information Society. We extend special thanks to the reviewers for their critical, thorough, and constructive feedback that resulted in improved versions of these articles. And of course, thank you to all the authors whose contributions are the result of an obvious dedication to improving access to all the information and services the web has to offer to the aging population.

\section{List of reviewers}

Shiri Azenkot, University of Washington, USA Jeffrey Bigham, University of Rochester, USA Jeremy Brudvik, Georgia Tech, USA

Anna Cavender, University of Washington, USA

Wendy Chisholm, University of Washington, USA

Rock Leung, University of British Columbia, Canada

Clayton Lewis, University of Colorado, USA

Karyn Moffatt, University of British Columbia, Canada Kristen Shinohara, University of Washington, USA 